\title{
Spherical aberration correction in multiphoton fluorescence imaging using objective correction collar
}

\author{
Wen Lo \\ Yen Sun \\ National Taiwan University \\ Department of Physics \\ Taipei 106, Taiwan \\ Sun-Jan Lin \\ National Taiwan University Hospital \\ Department of Dermatology \\ Taipei 106, Taiwan

\section{Shiou-Hwa Jee} \\ National Taiwan University Hospital \\ Department of Dermatology \\ Taipei 106, Taiwan \\ and \\ National Taiwan University College of Medicine \\ Department of Dermatology \\ Taipei 106, Taiwan

\section{Chen-Yuan Dong} \\ National Taiwan University \\ Department of Physics \\ Taipei 106, Taiwan
}

\begin{abstract}
Multiphoton microscopy has evolved into a powerful bioimaging tool in three dimensions. However, the ability to image biological specimens in-depth can be hindered by sample spherical aberration and scattering. These two phenomena can result in the degradation of image resolution and the loss of detected multiphoton signal. In this work, we use the correction collar (for cover glass thickness) associated with a water immersion objective in an attempt to improve multiphoton imaging. In the two samples we examined (human skin and rat tail tendon), we found that while the improvement in image resolution was not visible qualitatively, the measured axial fluorescence or second harmonic generation signal profiles indicate that the use of the correction collar can help to improve the detected multiphoton signals. The maximum increases are $36 \%$ and $57 \%$ for the skin (sulforhodamine B fluorescence) and tendon (second harmonic generation) specimens, respectively. Our result shows that for in-depth multiphoton imaging, the correction collar may be used to correct for spherical aberration. However, each tissue type needs to be examined to determine the optimal correction collar setting to be used. () 2005 Society of Photo-Optical Instrumentation Engineers.
\end{abstract} [DOI: $10.1117 / 1.1924614]$

Keywords: multiphoton; fluorescence; second harmonic generation; skin; rat tail tendon; spherical aberration; objective correction collar.

Paper 034009 received Dec. 1, 2003; revised manuscript received Sep. 7, 2004; accepted for publication Nov. 17, 2004; published online May 26, 2005.

\section{Introduction}

Since its introduction in 1990, multiphoton fluorescence microscopy has developed into be a powerful imaging technique for the acquisition of structural information of threedimensional biological samples. There are several major advantages associated with this imaging modality. First, imaging with the point-like, multiphoton excitation volume results in images with improved axial depth discrimination. An additional benefit associated with the limited excitation volume is the reduced sample photodamage. Furthermore, the nearinfrared excitation photons used for sample excitation are absorbed and scattered less than the ultraviolet or visible excitation sources used in one-photon excitation microscopy. ${ }^{1,2}$ The unique features of multiphoton fluorescence microscopy allow unique application of this technique in addressing a variety of bioimaging questions. Areas such as neurobiology, developmental biology, hepatology, drug delivery, and deeptissue imaging have all benefited from the development of multiphoton imaging techniques. ${ }^{3-9}$

Although multiphoton microscopy has proven to be a successful, in-depth, bioimaging method, limitations in its applications still exist. At increased imaging depths, optical phenomena such as refractive index mismatch induced spherical aberration and scattering can lead to resolution degradation

Address all correspondence to Chen Yuan Dong, National Taiwan University, Department of Physics, Taipei 106, Taiwan, Tel: 8862-2-3366-5155; Fax: 8862-2363-9984; E-mail: cydong@phys.ntu.edu.tw and the loss of fluorescence signal. While these conditions create inconveniences for qualitative multiphoton fluorescence microscopy, they can result in devastating effects for quantitative microscopy. In many areas of fluorescence microscopic applications, the ability to determine the absolute concentrations of fluorescence species is vital in providing a complete description of the phenomena being investigated. For example, in drug delivery studies, the quantification of fluorescently labeled drugs is important information for the development of improved drug delivery technologies. In transdermal drug delivery studies, multiphoton fluorescence microscopy has proven to be successful in revealing many aspects of the delivery process. However, the quantification of the fluorescent molecules in such studies remains elusive. ${ }^{6-8}$

\section{The Use of Objective Collar for Spherical Aberration Correction}

In the case of skin, the complex landscape of optical parameters proves to be the major hurdle in providing quantitative information of fluorescence phenomena in three dimensions. Previously, optical coherence tomography was used to measure the refractive indices and scattering coefficients of the skin. In terms of refractive indices, it was revealed that the surface stratum corneum of the skin has a refractive index of

1083-3668/2005/\$22.00 @ 2005 SPIE 


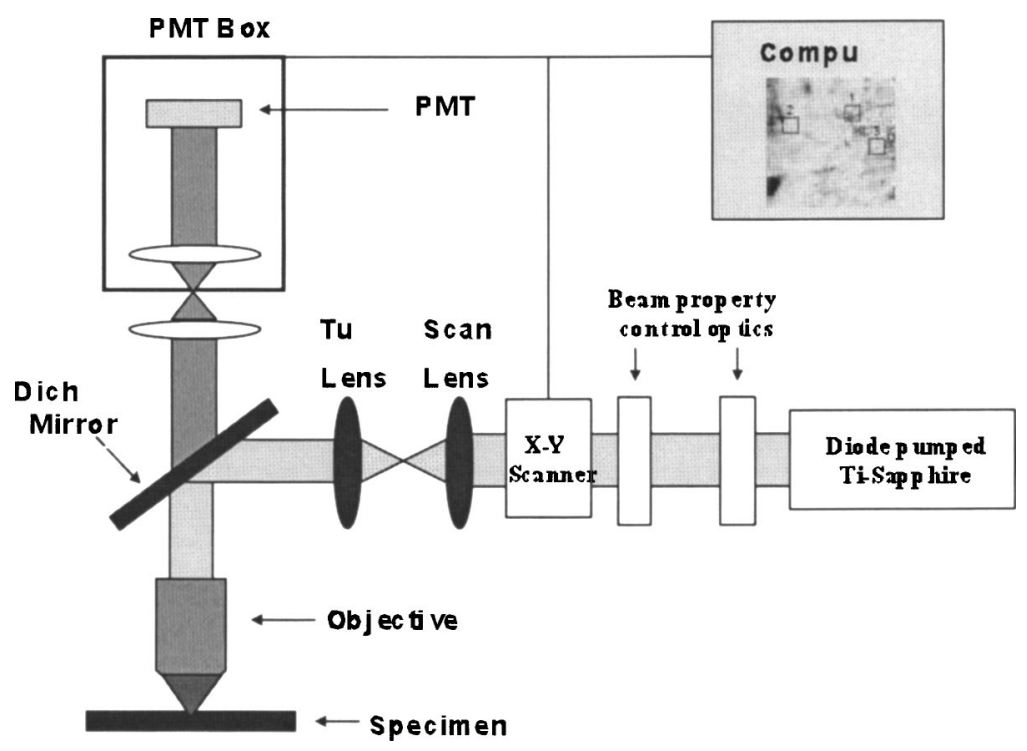

Fig. 1 An upright multiphoton fluorescence and SHG microscope.

1.47, a value close to that of typical immersion oil used for optical microscopy. In deeper epidermis, the index of refraction decreases to about 1.43 in the granular layer and is around 1.34 in basal layer. Beyond the epidermis, the index of refraction increases to about 1.41 in the upper dermis. The skin's scattering coefficients also vary significantly from the epidermis to the dermis. The stratum corneum has scattering coefficients around $1-1.5 \mathrm{~mm}^{-1}$ and the deeper granular layer's scattering coefficient is about $6-7 \mathrm{~mm}^{-1}$. At the basal layer, $4-5 \mathrm{~mm}^{-1}$ was the measured scattering coefficient and in the upper dermis, its value increases to approximately 5-8 $\mathrm{mm}^{-1} \cdot{ }^{10,11}$ The combined effects of refractive index mismatch and scattering result in difficulties in the understanding of microscopic image formation in the skin. While the imaging artifacts due to sample scattering are more difficult to correct for, different methods have been suggested in the literature to correct for refractive index mismatch induced spherical aberration. In the case of skin imaging, one possibility is to vary the settings for cover glass thickness correction collar to achieve improved imaging conditions. ${ }^{12,13}$ If effective, this approach may be the most convenient way to achieve image optimization for many biological specimens. However, to the best of our knowledge, such an approach has not yet been experimentally tested in skin imaging applications, and it is our intention to examine the effectiveness of such an approach in multiphoton tissue imaging. In addition to skin, extracellular matrix components such as collagen fibers are often targets of multiphoton imaging. ${ }^{14,15}$ Specifically, it is well known that collagen fibers can be induced to generate second harmonic signals. In addition, rat tail tendon collagen has a refractive index of approximately $1.5 .^{16}$ Therefore, it is conceivable that the use of the correction collar setting may help to optimize multiphoton imaging of rat tail tendon, a structure made primarily of second harmonic generation (SHG) producing collagen I fibers, and we will examine the possibility of using the correction collar in the optimization of SHG imaging of collagen fibers.

\section{Experimental Apparatus}

\subsection{Mulitphoton Fluorescence Microscope}

The multiphoton fluorescence microscope used in this study is similar to a system described previously. ${ }^{15}$ As shown in Fig. 1, a diode (Millenia X, Spectra Physics, Mountain View, CA) pumped titanium-sapphire laser (Tsunami, Spectra Physics) was used as the excitation source. 780 and $880 \mathrm{~nm}$ were the wavelengths used for fluorescence excitation and second harmonic generation from the sulforhodamine B (SRB) treated skin specimen and rat tail tendon, respectively. The output of the laser is guided towards our home-built microscope (E800, Nikon, Japan) using an $x-y$ mirror scanning system (model 6220, Cambridge Technology, Cambridge, MA). For optimal imaging, the excitation laser is beam expanded and reflected towards the water-immersion objective [Plan Apo, 60×, numerical aperature (NA) 1.2, Nikon] using a short-pass dichroic mirror (720DCSPXR, Chroma Technology, Brattleboro, VT). The power levels of 1.5 and $4.4 \mathrm{~mW}$ (at the samples) were used for the skin and rat tail tendon specimens, respectively. The fluorescence or second harmonic signal generated at the focal volume is collected by the objective in an epi-illuminated fashion. After passing through the dichroic mirror and an additional short pass filter (E680SP, Chroma Technology), the fluorescence is directed towards a photomultiplier tube (R7400P, Hamamatsu, Japan). Additional bandpass filters were used to isolate the fluorescence or SHG signal of interest. In the case of SRB treated skin specimen, a bandpass filter centered at $610 \mathrm{~nm}$ (QA610/75, Chroma Technology) was used to detect SRB fluorescence. For the collagen specimen, a different band pass filter centered at $440 \mathrm{~nm}$ (HQ440/20, Chroma Technology) was used to measure the SHG signal. In our single-photon counting detection mode, the signal photons are processed by a discriminator before being recorded by the image acquisition computer.

Central to this study is the cover glass thickness correction collar associated with the $60 \times$, Plan Apo objective. The cor- 


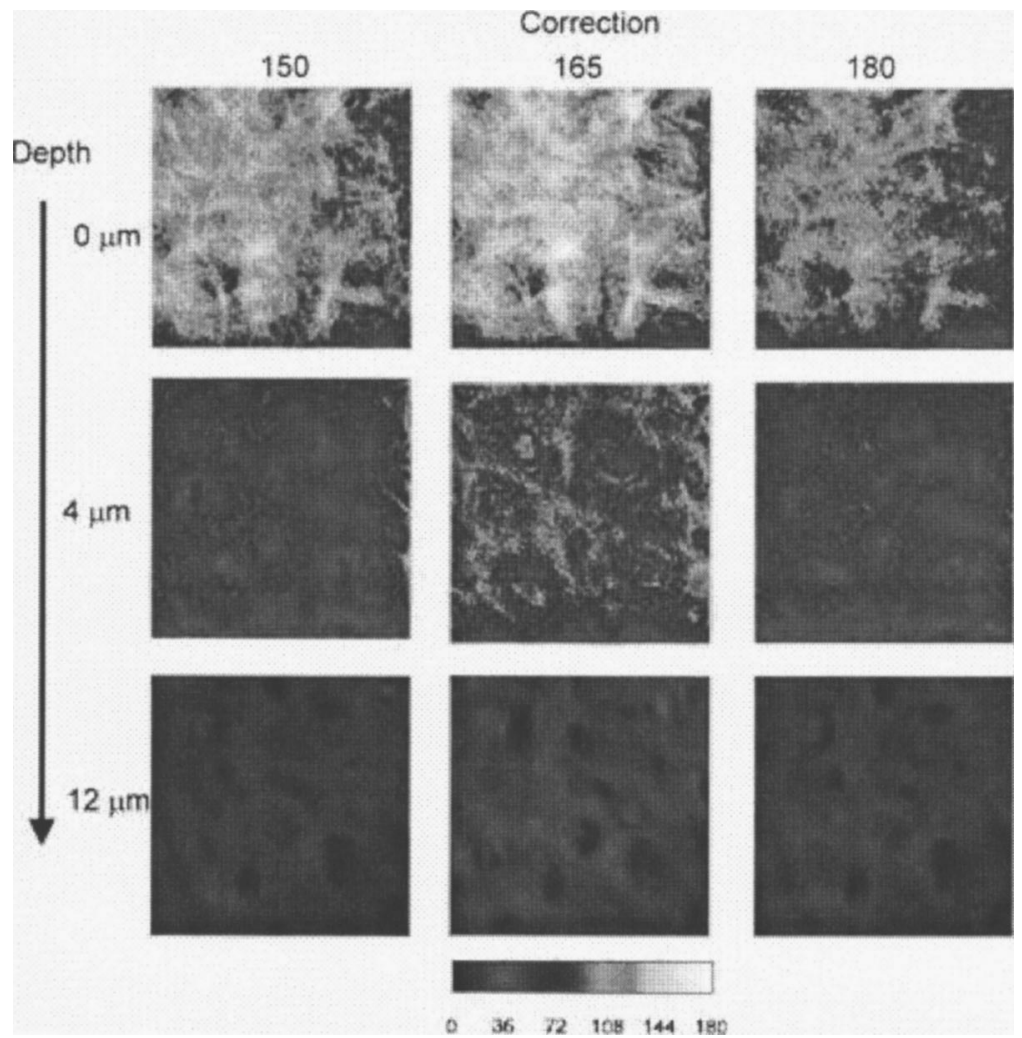

Fig. 2 Multiphoton images of SRB labeled skin at different objective (60× Plan Apo, NA 1.2, Nikon) correction collar settings (150, 165, and $180 \mu \mathrm{m})$.

rection collar is marked from 15 to 18 intended to correct for cover glasses with thickness from 150 to $180 \mu \mathrm{m}$. This range corrects for the No. 1 and No. 1.5 cover glasses commonly used for optical microscopic imaging. Since the refractive index of the skin surface and the rat tail tendon have refractive indices to be closer to that of typical immersion oil, we decided to use No. 1 cover glass for this investigation. ${ }^{10,11,16}$ With the proper correction collar setting, we can correct for the skin-induced spherical aberration by increasing the collar setting from 15. In our study, we acquired the images of the same area of fluorescently labeled skin at seven collar settings $(15,15.5,16,16.5,17,17.5$, and 18) without increasing the laser power.

For both the SRB skin and rat tail specimens, three sets of three-dimensional images were acquired and averaged for statistical analysis of the axial signal profiles. At the ends of experiments, we return to the starting correction collar setting to verify that the sample has not been photodamaged. We found that the power levels used for the two types of specimens did not cause significant photodamage during the course of the experiments. We also found that the experimental errors associated with averaging the three data sets at each collar setting were small in comparison with the measured signal values and we choose not to plot the standard deviations of the measurements in the averaged axial signal curves.

\subsection{Skin and Rat Tail Tendon Preparation}

The ex vivo human skin used was fluorescently labeled using sulforhodamine B, (SRB, S-1307, Molecular Probes, Eugene, OR). To prepare the labeling solution, polybutene sulfone buffer was mixed with ethanol in a 1:1 ratio. 5\% oleic acid was added to enhance the penetration of the SRB across the skin. The final labeling concentration of SRB was $0.5 \mathrm{mg} / \mathrm{mL}$. For SRB labeling, the ex vivo skin was immersed in the SRB solution for $24 \mathrm{~h}$ prior to being removed for imaging purposes. The tendon specimen can be easily obtained by removing the tendon from the excised tail of a rat.

\section{Results and Discussions}

To compare the performances of the fluorescence skin images under different degrees of correction settings, we show the cross-sectional images of the skin at different depths $(0,4$, and $12 \mu \mathrm{m})$ in Fig. 2. Since the skin structure can be heterogeneous, we define the surface or the $0 \mu \mathrm{m}$ position at the axial position at which the average fluorescence of the crosssectional image is a maximum. In Fig. 2, we only displayed the skin images at three correction collar settings of $15,16.5$, and 18, corresponding to the equivalent correction for cover glass with thickness of 150,165 , and $180 \mu \mathrm{m}$, respectively. The images at each correction collar setting are displayed at the same intensity scale (as indicated by the color palette). Visual examination of Fig. 2 shows that it is difficult to discern differences of image resolution under the different collar settings. However, changes of the SRB fluorescence intensity with imaging depths and correction collar settings shown in Fig. 4 indicate the quantitative differences between the different collar settings. The detailed comparison of the axial fluorescence intensity profiles was done by plotting the average fluorescence intensity at different collar settings. As shown in 


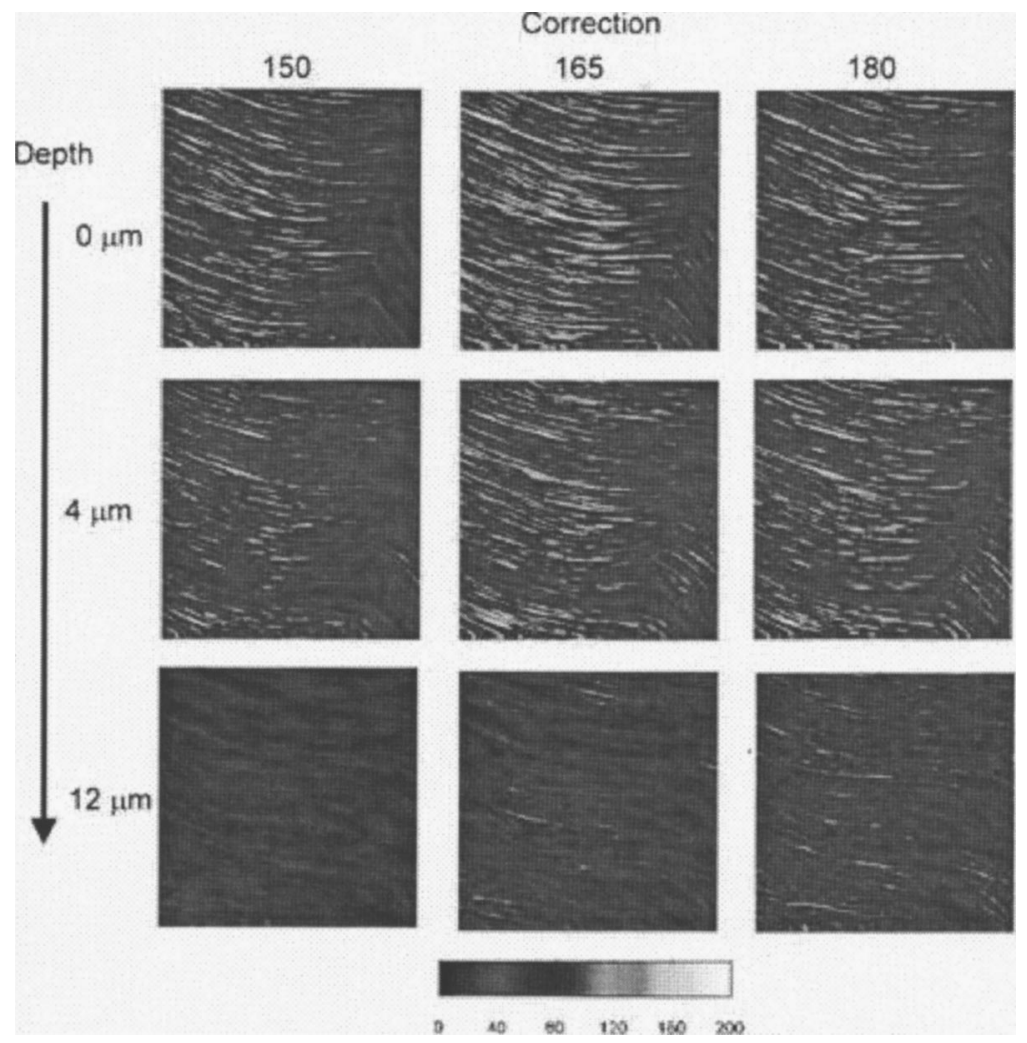

Fig. 3 SHG images (centered at $440 \mathrm{~nm}$ ) of rat tail tendon at three different objective (60× Plan Apo, NA 1.2, Nikon) correction collar settings (150, 165 , and $180 \mu \mathrm{m})$.

Fig. 4, we plotted the absolute values of the average fluorescence intensity at depths ranging from 0 to $28 \mu \mathrm{m}$ for all collar correction settings between 150 and $180 \mu \mathrm{m}$. Visual examination shows that the average fluorescence intensity at each imaging depth varies with the collar setting. The variation (for depths up to $28 \mu \mathrm{m})$ can be as large as $36 \%(0 \mu \mathrm{m}$ data). Another interesting observation from Fig. 4 is that the average fluorescence tends to peak around the cover glass correction of $165 \mu \mathrm{m}$ for the imaging depths of $0,4,8,12$, and $16 \mu \mathrm{m}$. This is an indication that by increasing the collar setting, we are compensating for the spherical aberration induced by the surface skin layers. However, over correction (beyond $165 \mu \mathrm{m}$ of cover glass thickness) actually lead to a decrease in measured fluorescence intensity, indicating a degradation in the multiphoton PSF responsible for generating the fluorescence signal. However, at deeper imaging depths of 20, 24 , and $28 \mu \mathrm{m}$, the measured fluorescence intensities show little dependence on the collar setting. This observation implies that the complicated refractive index landscape of the skin render a decrease in the effectiveness of the correction collar in compensating for the refractive indices of surface skin layers. $^{10,11}$

We performed similar experiments on rat tail tendon and the SHG images of the tendon specimen as functions of varying depths and correction collar settings are shown in Fig. 3. In this case, we also displayed the SHG images at the depths of 0,4 , and $12 \mu \mathrm{m}$ with the correction collar settings for 150 , 165, and $180 \mu \mathrm{m}$ cover glass thicknesses. As in the case of SRB treated fluorescence images, it is difficult to assess the changes in resolution by varying the collar settings. In addi- tion, the SHG axial profiles are computed and plotted in Fig. 4 in manners similar to the SRB skin data. Figure 4 shows that the rat tail tendon result demonstrates a different dependence compared to the SRB skin data. In the case of rat tail tendon, there can be an increase in the SHG signal at depth along with an increase in the correction collar settings. The increase can be as large as $57 \%$ (imaging depth of $28 \mu \mathrm{m}$ ). At the depths of 0,4 , and $8 \mu \mathrm{m}$, the SHG signal increases until the correction collar setting for $170 \mu \mathrm{m}$ cover glass and decreases. However, at greater imaging depths of 12, 16, 20, 24, and $28 \mu \mathrm{m}$, the SHG intensity displays a different behavior with the increase in correction collar settings. At the imaging depths of 16 and $20 \mu \mathrm{m}$, the increase in SHG signal plateaus at the correction collar setting of $175 \mu \mathrm{m}$. However, at 24 and $28 \mu \mathrm{m}$ deep, the SHG signal increases up to the collar setting of $180 \mu \mathrm{m}$. These observations are consistent if the refractive index of the region of the rat tail tendon being examined is relative consistent. At shallower depths $(0,4$, and $8 \mu \mathrm{m})$, the increase of the collar settings initially helps in increasing the measured SHG signal. The drop in SHG signal beyond the collar setting of $170 \mu \mathrm{m}$ shows that over correction actually lead to a PSF degradation that lead to less efficient SHG. As the imaging depths increase, this effect tends to be less obvious as higher correction collar settings are needed to correct for the additional tendon tissue the excitation beam needs to traverse in reaching the focal point.

\section{Conclusions}

In this work, we demonstrated the use of the cover glass collar of a water immersion objective in the correction for index 
Lo et al.: Spherical aberration correction ...
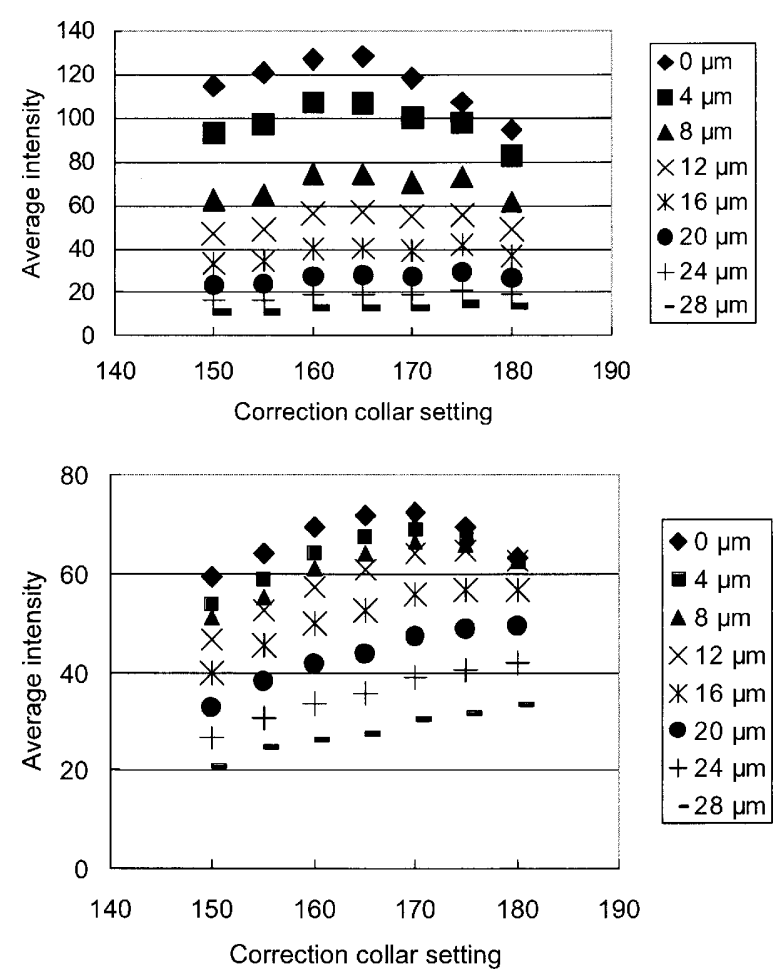

Fig. 4 Average fluorescence signal in SRB treated skin (top) and SHG signal in rat tail tendon (bottom) at different objective collar correction settings (60× Plan Apo, NA 1.2, Nikon) at different depths ( $\mu \mathrm{m})$.

mismatch induced spherical aberration in multiphoton imaging biological tissues such as the skin and rat tail tendon. We found that in the case of skin, the correction collar can help to obtain improved SRB fluorescence measurement by compensating for the refractive index of the skin. However, the ability of the correction collar to compensate for the skin's refractive index decrease with increasing imaging depths, most likely due to the complex landscape of the skin's refractive index profiles. In the case of a rat tail tendon, a specimen with a more uniform refractive index, the correction collar is effective in improving the measured SHG signal up to $28 \mu \mathrm{m}$. Our observations demonstrate that the correction collar settings can be used to correct for the spherical aberration caused by a mismatch in refractive indices. However, since biological tissue specimens can have a wide range of refractive indices properties, the entire range of correction collar settings need to be explored for the tissue type of interest prior to determine the optimal collar setting to be used.

\section{Acknowledgments}

The authors would like to acknowledge the support of NSC 92-2112-M-002-018 and NSC 92-3112-B-002-048.

\section{References}

1. W. Denk, J. H. Strickler, and W. W. Webb, "2-photon laser scanning fluorescence microscopy," Science 248(4951), 73-76 (1990).

2. P. T. C. So, C. Y. Dong, B. R. Masters, and K. M. Berland, "Twophoton excitation fluorescence microscopy," Annual Review of Biomedical Engineering 2, 399-429 (2000).

3. W. Denk, M. Sugimori, and R. Llinas, "2 types of calcium response limited to single spines in cerebellar purkinje-cells," Proc. Natl. Acad. Sci. U.S.A. 92(18), 8279-8282 (1995).

4. J. M. Squirrell, D. L. Wokosin, J. G. White, and B. D. Bavister, "Long-term two-photon fluorescence imaging of mammalian embryos without compromising viability," Nat. Biotechnol. 17(8), 763767 (1999).

5. H. S. Lee, Y. Liu, H. C. Chen, L. L. Chiou, G. T. Huang, W. Lo, and C. Y. Dong, "Optical biopsy of liver fibrosis using multiphoton microscopy," Opt. Lett. 29(22), 2614-2616 (2004).

6. B. Yu, C. Y. Dong, P. T. C. So, D. Blankschtein, and R. Langer, "In vitro visualization and quantification of oleic acid induced changes in transdermal transport using two-photon fluorescence microscopy," $J$. Invest. Dermatol. 117(1), 16-25 (2001).

7. B. Yu, K. H. Kim, P. T. C. So, D. Blankschtein, and R. Langer, "Topographic heterogeneity in transdermal transport revealed by high-speed two-photon microscopy: Determination of representative skin sample sizes," J. Invest. Dermatol. 118(6), 1085-1088 (2002).

8. B. Yu, K. H. Kim, P. T. C. So, D. Blankschtein, and R. Langer, "Visualization of oleic acid-induced transdermal diffusion pathways using two-photon fluorescence microscopy," J. Invest. Dermatol. 120(3), 448-455 (2003).

9. P. T. C. So, H. Kim, and I. E. Kochevar, "Two-photon deep tissue ex vivo imaging of mouse dermal and subcutaneous structures," Opt. Express 3(9), 339-350 (1998).

10. G. J. Tearney, M. E. Brezinski, J. F. Southern, B. E. Bouma, M. R. Hee, and J. G. Fujimoto, "Determination of the refractive-index of highly scattering human tissue by optical coherence tomography," Opt. Lett. 20(21), 2258-2260 (1995).

11. A. Knuttel and M. Boehlau-Godau, "Spatially confined and temporally resolved refractive index and scattering evaluation in human skin performed with optical coherence tomography," J. Biomed. Opt. 5(1), 83-92 (2000).

12. M. J. Booth, M. A. A. Neil, and T. Wilson, "Aberration correction for confocal imaging in refractive-index-mismatched media," Journal of Microscopy-Oxford 192, 90-98 (1998).

13. M. J. Booth and T. Wilson, "Strategies for the compensation of specimen-induced spherical aberration in confocal microscopy of skin," Journal of Microscopy-Oxford 200, 68-74 (2000).

14. C. K. Tung, Y. Sun, W. Lo, S. J. Lin, S. H. Jee, and C. Y. Dong, "Effects of objective numerical apertures on achievable imaging depths in multiphoton microscopy," (unpublished).

15. Y. Sun, J. W. Su, W. Lo, S. J. Lin, S. H. Jee, and C. Y. Dong, "Multiphoton polarization imaging of the stratum corneum and the dermis in ex-vivo human skin," Opt. Express 11(25), 3377-3384 (2003).

16. P. Stoller, P. M. Celliers, K. M. Reiser, and A. M. Rubenchik, "Quantitative second-harmonic generation microscopy in collagen," Appl. Opt. 42(25), 5209-5219 (2003). 